\title{
A Revisit on the Derivation of the Particular Solution for the Differential Operator $\Delta^{2} \pm \lambda^{2}$
}

\author{
Guangming Yao ${ }^{1}$, C. S. Chen ${ }^{1, *}$ and Chia Cheng Tsai ${ }^{2}$ \\ ${ }^{1}$ Department of Mathematics, University of Southern Mississippi, Hattiesburg, MS \\ 39406, USA \\ 2 Department of Marine Environment Engineering, National Kaohsiung Marine \\ University, Taiwan
}

Received 28 February 2009; Accepted (in revised version) 25 August 2009

Available online 18 November 2009

\begin{abstract}
In this paper, we applied the polyharmonic splines as the basis functions to derive particular solutions for the differential operator $\Delta^{2} \pm \lambda^{2}$. Similar to the derivation of fundamental solutions, it is non-trivial to derive particular solutions for higher order differential operators. In this paper, we provide a simple algebraic factorization approach to derive particular solutions for these types of differential operators in 2D and 3D. The main focus of this paper is its simplicity in the sense that minimal mathematical background is required for numerically solving higher order partial differential equations such as thin plate vibration. Three numerical examples in both 2D and 3D are given to validate particular solutions we derived.
\end{abstract}

AMS subject classifications: 35J05, 35J25, 65D05, 65D15

Key words: The method of fundamental solutions, radial basis functions, meshless methods, polyharmonic splines, the method of particular solutions.

\section{Introduction}

The idea of splitting a given partial differential equation into solving a homogeneous equation and an inhomogeneous equation is well known. In recently years, such approach becomes very popular for various boundary meshless methods such as the Trefftz method, the method of fundamental solutions $[8,9]$, and the boundary knot method (BKM) [4], etc. By evaluating the particular solution, these boundary meshless methods can be extended from solving only homogeneous equations to inhomogeneous equations and time-dependent problems [1,9]. As a consequence, many

\footnotetext{
*Corresponding author.

URL: http://www.math.usm.edu/cschen/

Email: guangmingyao@gmail.com (G. Yao), cschen.math@gmail.com (C. S. Chen), tsaichiacheng@mail. nkmu.edu.tw (C. C. Tsai)
} 
numerical techniques have been developed to evaluate particular solutions for various types of partial differential equations. Chen and Rashed [2] were first to extend the derivation of particular solutions to Helmholtz-type equations using thin plate splines. Muleshkov et al. [11] further extended the concept to polyharmonic splines. However, the derivation of particular solutions using the annihilator method and algebraic techniques in [11] were too tedious to use for solving complicated differential operators. Cheng [6] revisited the problem using the technique of fundamental solutions so that particular solutions can be easily derived. Recently, Muleshkov and Golberg [12], and Chen et al. [3] derived particular solution for more complicate differential operators using radial basis functions and Chebyshev polynomials. In 2009, Tsai et al. [13] extended the derivations of particular solutions to polyharmonic, polyHelmholtz operators and their products.

In contrast to the tedious derivation of particular solutions for Helmholtz-type differential operators shown in [11] and its extension to general operators [13], we propose a simple algebraic factorization approach to derive particular solutions for the differential operators

$$
\Delta^{2} \pm \lambda^{2}
$$

in 2D [5] and 3D using polyharmonic splines. On the other hand, Young et al. [14] solved the homogeneous equation of plate vibration problem in which $\Delta^{2}-\lambda^{2}$ is the differential operator. Coupled with the particular solutions derived in this paper, [14] can be effectively extened to solving the arbitrarily loaded flexural vibrations of an uniform thin plate.

This paper is organized as follows. In section 2, we derive particular solutions for polyharmonic splines which includes two dimensional and three dimensional cases. In Section 3, we derive particular solutions for the monomial term for $\Delta^{2}-\lambda^{2}$. In Section 4, numerical examples for two 2D examples and one 3D example are given. In Section 5, we conclude this paper with opening issues and future applications.

\section{Particular solutions for polyharmonic splines}

Let us consider the following boundary value problem

$$
\begin{array}{ll}
\left(\Delta^{2}-\lambda^{2}\right) u=f(\mathbf{x}), & \mathbf{x} \in \Omega \subset \mathbb{R}^{d}, \\
\mathcal{B}_{1} u=g(\mathbf{x}), & \mathbf{x} \in \partial \Omega, \\
\mathcal{B}_{2} u=h(\mathbf{x}), & \mathbf{x} \in \partial \Omega,
\end{array}
$$

where $\lambda$ is a non-zero constant, $\Delta$ is the Laplacian, $\mathcal{B}_{1}$ and $\mathcal{B}_{2}$ are the boundary differential operators, $f, g$ and $h$ are given functions, and $\Omega$ is an open bounded domain in $\mathbb{R}^{d}, d=2,3$, with boundary $\partial \Omega$. Note that $\mathbf{x}=(x, y)$ in $2 \mathrm{D}$ and $\mathbf{x}=(x, y, z)$ in $3 \mathrm{D}$. For $d=2$, (2.1)-(2.3) govern the loaded flexural vibrations of a uniform thin plate.

Let $u_{p}$ be a particular solution of the governing equation, then it satisfies

$$
\left(\Delta^{2}-\lambda^{2}\right) u_{p}=f(\mathbf{x}),
$$


but does not necessarily satisfy the boundary condition. If $u_{p}$ in (2.4) can be obtained, then (2.1) -(2.3) can be reduced to the following homogeneous equation through the variable substitution $w=u-u_{p}$, i.e.,

$$
\begin{array}{ll}
\left(\Delta^{2}-\lambda^{2}\right) w=0, & \mathbf{x} \in \Omega, \\
\mathcal{B} w=g(\mathbf{x})-u_{p}, & \mathbf{x} \in \partial \Omega .
\end{array}
$$

The above homogeneous equation can be easily solved using boundary methods such as the Trefftz method [1], the method of fundamental solutions [8,9], and the boundary knot method (BKM) [4], etc. The key problem to be considered is how to obtain an approximation to the particular solution $u_{p}$.

For arbitrary function $f(\mathbf{x})$, it is difficult, if not impossible, to obtain $u_{p}$. Therefore, it is essential to approximate the source term $f(\mathbf{x})$ by a series of basis functions [9]. In this section, we focus on the derivation of the closed-form approximate particular solution of (2.4).

Let $P_{Q-1}^{d}$ denote the space spanned by all $d$-variate polynomials of degree up to $Q-1$ and pick a basis $\left\{p_{\ell}\right\}_{\ell=1}^{q}$ of this space. The dimension of $q$ is

$$
\left(\begin{array}{c}
Q-1+d \\
d
\end{array}\right)
$$

To find approximate particular solutions $u_{p}$ to

$$
\left(\Delta^{2}-\lambda^{2}\right) u_{p}=f(\mathbf{x}),
$$

we approximate $f$ by $\hat{f}$ as follows

$$
\hat{f}(\mathbf{x})=\sum_{j=1}^{m} \alpha_{j} \phi\left(r_{j}\right)+\sum_{\ell=1}^{q} \beta_{\ell} p_{\ell}(\mathbf{x}),
$$

where $r_{j}=\left\|\mathbf{x}-\mathbf{x}_{j}\right\|,\left\{\mathbf{x}_{j}\right\}_{1}^{m}$ is a unisolvent set of points for polynomial interpolation. $\varphi$ in (2.8) is radial basis functions. The coefficients $\left\{\alpha_{j}\right\}$ and $\left\{\beta_{\ell}\right\}$ can be determined by interpolating $f$ by $\hat{f}$ on $\left\{\mathbf{x}_{j}\right\}_{1}^{m}$. The following additional conditions for (2.8) are required

$$
\sum_{j=1}^{m} \alpha_{j} p_{\ell}(\mathbf{x})=0, \quad 1 \leq \ell \leq q .
$$

As was shown by Duchon [7], (2.8)-(2.9) has a unique solution. Then, the approximate particular solution can be obtained in the following way

$$
u_{p}(\mathbf{x}) \simeq \hat{u}_{p}(\mathbf{x})=\sum_{j=1}^{m} a_{j} \Phi\left(r_{j}\right)+\sum_{\ell=1}^{q} \beta_{\ell} \chi_{\ell}(\mathbf{x}),
$$


where

$$
\begin{aligned}
& \left(\Delta^{2}-\lambda^{2}\right) \Phi\left(r_{j}\right)=\phi\left(r_{j}\right), \\
& \left(\Delta^{2}-\lambda^{2}\right) \chi_{\ell}=p_{\ell} .
\end{aligned}
$$

In this paper we apply the following polyharmonic splines to approximate $f(\mathbf{x})$ in (2.7)

$$
\phi(r):=\varphi^{(n)}(r)=\left\{\begin{array}{ll}
r^{2 n} \ln r, & \text { in } \mathbb{R}^{2}, \\
r^{2 n-1,} & \text { in } \mathbb{R}^{3},
\end{array} \quad n \geq 1 .\right.
$$

The reason that we choose $\phi$ to be polyharmonic splines is due to its unique property that $\Delta^{L} r^{2 n} \ln (r)=0$ for $L$ sufficiently large [11].

\subsection{Two dimensional case}

In this subsection, we consider the derivation of the approximate particular solution of the following equation in $2 \mathrm{D}$

$$
\left(\Delta^{2}-\lambda^{2}\right) \Phi^{(n)}(r)=r^{2 n} \ln r
$$

where $\lambda>0, n$ is a positive integer and $\Delta$ is the Laplacian where

$$
\Delta=\frac{1}{r} \frac{d}{d r}\left(r \frac{d}{d r}\right) .
$$

For illustration of basic idea, let us first consider the case when $n=2$. We observe that for $r \neq 0$ and $k \geq 3$

$$
\Delta r^{4} \ln r=16 r^{2} \ln r+8 r^{2}, \quad \Delta^{2} r^{4} \ln r=64 \ln r+96, \quad \Delta^{k} r^{4} \ln r=0 .
$$

Since $\Delta^{4} r^{4} \ln r=0$, it follows that

$$
\left(1-\left(\frac{\Delta^{2}}{\lambda^{2}}\right)^{2}\right) r^{4} \ln r=r^{4} \ln r
$$

The differential operator on the the left hand side of (2.15) can be factored as follows:

$$
\left(1-\frac{\Delta^{2}}{\lambda^{2}}\right)\left(1+\frac{\Delta^{2}}{\lambda^{2}}\right) r^{4} \ln r=r^{4} \ln r
$$

By rearranging the above equation, we have

$$
\left(\Delta^{2}-\lambda^{2}\right) \underbrace{\left(-\frac{1}{\lambda^{2}}\right)\left(1+\frac{\Delta^{2}}{\lambda^{2}}\right) r^{4} \ln r}_{\Phi^{(2)}(r)}=r^{4} \ln r .
$$


Comparing (2.13) and (2.17), we can easily see the underbrace in (2.17) is a particular solution $\Phi^{(2)}$, i.e.,

$$
\begin{aligned}
\Phi^{(2)}(r) & =\left(-\frac{1}{\lambda^{2}}\right)\left(1+\frac{\Delta^{2}}{\lambda^{2}}\right) r^{4} \ln r \\
& =-\frac{r^{4} \ln r}{\lambda^{2}}-\frac{64 \ln r+96}{\lambda^{4}}, \quad \text { for } r \neq 0 .
\end{aligned}
$$

However, we notice that $\Phi^{(2)}(r)$ in (2.18) has a singularity at $r=0$, and the normal derivative of $\Phi^{(2)}(r)$ also has singularity term $1 / r^{2}$ at $r=0$ as follows

$$
\frac{1}{r} \frac{\partial \Phi^{(2)}(r)}{\partial r}=-\frac{r^{2}(4 \ln r+1)}{\lambda^{2}}-\frac{64}{\lambda^{4} r^{2}} .
$$

To remove the singularities, we use a similar technique to the one shown in [8]. We denote $K_{0}(\sqrt{\lambda} r)$ and $Y_{0}(\sqrt{\lambda} r)$ as the first kind and second kind of Bessel functions with order 0 , respectively. For $r \neq 0$, we have

$$
(\Delta-\lambda) K_{0}(\sqrt{\lambda} r)=0, \quad(\Delta+\lambda) Y_{0}(\sqrt{\lambda} r)=0,
$$

which implies

$$
\begin{aligned}
& \left(\Delta^{2}-\lambda^{2}\right) K_{0}(\sqrt{\lambda} r)=(\Delta+\lambda)(\Delta-\lambda) K_{0}(\sqrt{\lambda} r)=0, \\
& \left(\Delta^{2}-\lambda^{2}\right) Y_{0}(\sqrt{\lambda} r)=(\Delta-\lambda)(\Delta+\lambda) Y_{0}(\sqrt{\lambda} r)=0 .
\end{aligned}
$$

We also notice that [10]

$$
\begin{aligned}
& K_{0}(\sqrt{\lambda} r)=-\gamma-\ln \left(\frac{\sqrt{\lambda}}{2}\right)-\ln r+\mathcal{O}(r), \\
& Y_{0}(\sqrt{\lambda} r)=\frac{2}{\pi}\left(\gamma+\ln \left(\frac{\sqrt{\lambda}}{2}\right)+\ln r\right)+\mathcal{O}(r),
\end{aligned}
$$

where $\gamma \simeq 0.5772156649015328$ is the Euler's constant. We observe that $K_{0}$ and $Y_{0}$ contain $\ln r$ which can be used to remove the singularities in (2.18) and (2.19). Let

$$
\Phi^{(2)}(r)=-\frac{r^{4} \ln r}{\lambda^{2}}-\frac{64 \ln r+96}{\lambda^{4}}+a K_{0}(\sqrt{\lambda} r)+b Y_{0}(\sqrt{\lambda} r)
$$

Then,

$$
\frac{1}{r} \frac{\partial \Phi^{(2)}(r)}{\partial r}=-\frac{r^{2}}{\lambda^{2}}(4 \ln r+1)-\frac{64}{\lambda^{4} r^{2}}-\frac{a}{r} \sqrt{\lambda} K_{1}(\sqrt{\lambda} r)-\frac{b}{r} \sqrt{\lambda} Y_{1}(\sqrt{\lambda} r),
$$

where $K_{1}$ and $Y_{1}$ [10] are the first and second kind of Bessel functions with order 1, respectively. To remove the singularity of $\ln r$ in (2.22), we have

$$
-\frac{64}{\lambda^{4}}+a(-1)+b\left(\frac{2}{\pi}\right)=0 .
$$


To remove the singularities of $1 / r^{2}$ and $\ln r$ in (2.23), since

$$
\begin{aligned}
& -\frac{\sqrt{\lambda} K_{1}(\sqrt{\lambda} r)}{r}=\frac{\lambda}{4}-\frac{\lambda \gamma}{2}-\frac{\lambda}{2} \ln \left(\frac{\sqrt{\lambda}}{2}\right)-\frac{\lambda}{2} \ln r-\frac{1}{r^{2}}+\mathcal{O}(r) \\
& -\frac{\sqrt{\lambda} Y_{1}(\sqrt{\lambda} r)}{r}=-\frac{2}{\pi}\left[-\frac{\lambda}{4}+\frac{\lambda \gamma}{2}+\frac{\lambda}{2} \ln \left(\frac{\sqrt{\lambda}}{2}\right)+\frac{\lambda}{2} \ln r-\frac{1}{r^{2}}\right]+\mathcal{O}(r),
\end{aligned}
$$

we have that

$$
-\frac{64}{\lambda^{4}}+a(-1)+b\left(\frac{2}{\pi}\right)=0
$$

and

$$
a\left(-\frac{\lambda}{2}\right)+b\left(-\frac{2}{\pi}\right)\left(\frac{\lambda}{2}\right)=0
$$

Notes that (2.24) and (2.27) are identical. This implies that

$$
a=-\frac{32}{\lambda^{4}}, \quad \text { and } \quad b=\frac{16 \pi}{\lambda^{4}} .
$$

Thus,

$$
\Phi^{(2)}(r)= \begin{cases}-\frac{r^{4} \ln r}{\lambda^{2}}-\frac{64 \ln r+96}{\lambda^{4}}-\frac{16}{\lambda^{4}}\left(2 K_{0}(\sqrt{\lambda} r)-\pi Y_{0}(\sqrt{\lambda} r)\right), & \text { for } r \neq 0, \\ \frac{64}{\lambda^{4}}\left(\gamma+\ln \left(\frac{\sqrt{\lambda}}{2}\right)\right)-\frac{96}{\lambda^{4}}, & \text { for } r=0 .\end{cases}
$$

Note that [10]

$$
\frac{d K_{0}(\sqrt{\lambda} r)}{d r}=-\sqrt{\lambda} K_{1}(\sqrt{\lambda} r), \quad \frac{d Y_{0}(\sqrt{\lambda} r)}{d r}=-\sqrt{\lambda} Y_{1}(\sqrt{\lambda} r) .
$$

By taking derivative of $\Phi^{(2)}$ in (2.29) directly, we have

$$
\begin{aligned}
& \frac{1}{r} \frac{\partial \Phi^{(2)}(r)}{\partial r}=-\frac{r^{2}}{\lambda^{2}}(4 \ln r+1)-\frac{64}{\lambda^{4} r^{2}} \\
& +\frac{16 \sqrt{\lambda}}{\lambda^{4} r}\left(2 K_{1}(\sqrt{\lambda} r)-\pi Y_{1}(\sqrt{\lambda} r)\right), \quad \text { for } r \neq 0, \\
& \frac{1}{r} \frac{\partial \Phi^{(2)}(r)}{\partial r}=0, \\
& \text { for } r=0 \text {. }
\end{aligned}
$$

Using the following identities [10]

$$
\begin{aligned}
& \frac{d\left(K_{1}(\sqrt{\lambda} r)\right)}{d r}=-\sqrt{\lambda} K_{0}(\sqrt{\lambda} r)-\frac{1}{r} K_{1}(\sqrt{\lambda} r), \\
& \frac{d\left(Y_{1}(\sqrt{\lambda} r)\right)}{d r}=\sqrt{\lambda} Y_{0}(\sqrt{\lambda} r)-\frac{1}{r} Y_{1}(\sqrt{\lambda} r),
\end{aligned}
$$


we can easily obtain the following

$$
\Delta \Phi^{(2)}(r)= \begin{cases}-\frac{8 r^{2}}{\lambda^{2}}(2 \ln r+1)-\frac{16}{\lambda^{3}}\left(2 K_{0}(\sqrt{\lambda} r)+\pi Y_{0}(\sqrt{\lambda} r)\right), & \text { for } r \neq 0 \\ 0, & \text { for } r=0\end{cases}
$$

To find the general form of particular solutions $\Phi^{(n)}(r)$, we can follow the same procedure as shown above. We start with the following identity

$$
\left(1-\left(\frac{\Delta^{2}}{\lambda^{2}}\right)^{m+1}\right) r^{2 n} \ln r=r^{2 n} \ln r, \quad m=\left[\frac{n}{2}\right],
$$

where $m$ means the largest integer not greater than $n / 2$, and

$$
\Delta^{k} r^{2 n} \ln r=0, \quad \text { if } k \geq m+1 .
$$

Similarly, (2.34) can be written as follows

$$
\left(\Delta^{2}-\lambda^{2}\right)\left(\frac{-1}{\lambda^{2}}\right) \sum_{i=0}^{m}\left(\frac{\Delta^{2}}{\lambda^{2}}\right)^{i} r^{2 n} \ln r=r^{2 n} \ln r .
$$

It follows that

$$
\Phi^{(n)}(r)=-\frac{1}{\lambda^{2}} \sum_{i=0}^{m}\left(\frac{\Delta^{2}}{\lambda^{2}}\right)^{i} r^{2 n} \ln r, \quad m=\left[\frac{n}{2}\right] .
$$

The explicit form of $\Phi^{(n)}(r)$ in (2.36) can be obtained easily by direct differentiation or using the symbolic software such as Mathematica or Maple.

For $n$ even, a singularity will appear in $\Delta^{2 m} r^{2 n} \ln r$ and must be removeed as in the $r^{4} \ln r$ case. For $n$ odd, there is no singularity in $\Phi^{(n)}(r)$, but the normal derivative of $\Phi^{(n)}(r)$ will have a singularity which also comes from

$$
\frac{1}{r} \frac{\partial \Delta^{2 m} r^{2 n} \ln r}{\partial r} .
$$

It follows that for $r \geq 0$,

$$
\begin{aligned}
\Phi^{(n)}(r)= & -\frac{1}{\lambda^{2}} \sum_{i=0}^{m}\left(\frac{\Delta^{2}}{\lambda^{2}}\right)^{i} r^{2 n} \ln r \\
& -\frac{4^{n-1}(n !)^{2}}{\lambda^{n+2}}\left(2 K_{0}(\sqrt{\lambda} r)+(-1)^{n+1} \pi Y_{0}(\sqrt{\lambda} r)\right),
\end{aligned}
$$

and

$$
\Phi^{(n)}(0)=\lim _{r \rightarrow 0} \Phi^{(n)}(r) .
$$

In Appendix, we provide the explicit particular solutions and their derivatives for $n=3,4$ in $2 \mathrm{D}$ and $3 \mathrm{D}$ cases. 
If $\left(\Delta^{2}-\lambda^{2}\right)$ in $(2.13)$ is replaced by $\left(\Delta^{2}+\lambda^{2}\right)$, we can obtain a similar result as follows:

$$
\begin{aligned}
\Phi^{(n)}=\frac{1}{\lambda^{2}} & \sum_{i=0}^{m}\left(-\frac{\Delta^{2}}{\lambda^{2}}\right)^{i} r^{2 n} \ln r \\
& +\frac{4^{n-1}(-1)^{m}(n !)^{2}}{\lambda^{n+2}}\left(2 K_{0}(\sqrt{\lambda} r)+(-1)^{n+1} \pi Y_{0}(\sqrt{\lambda} r)\right)
\end{aligned}
$$

\subsection{Three dimensional case}

A similar approach to the one seen above can be applied to find the closed form $\Phi^{(n)}(r)$ in (2.13) in the three-dimensional case. We consider

$$
\left(\Delta^{2}-\lambda^{2}\right) \Phi^{(n)}(r)=r^{2 n-1}, \quad n=1,2,3, \cdots .
$$

In $3 D$, the Laplacian is denoted as follows:

$$
\Delta=\frac{1}{r^{2}} \frac{d}{d r}\left(r^{2} \frac{d}{d r}\right)
$$

When $n=1$, it is easy to show that

$$
\Phi^{(1)}(r)= \begin{cases}-\frac{r}{\lambda^{2}}+\frac{1}{\lambda^{3} r}\left(e^{-\sqrt{\lambda} r}-\cos (\sqrt{\lambda} r)\right), & \text { for } r \neq 0 \\ -\lambda^{\frac{-5}{2}}, & \text { for } r=0 .\end{cases}
$$

Again, we first consider the case for $n=2$. We observe that for $r \neq 0$

$$
\Delta r^{3}=12 r, \quad \Delta^{2} r^{3}=\frac{24}{r}, \quad \Delta^{k} r^{3}=0, \quad \text { for } k \geq 3 .
$$

We start with the following identity

$$
\left(1-\left(\frac{\Delta^{2}}{\lambda^{2}}\right)^{2}\right) r^{3}=r^{3}
$$

Similar to the 2D case, the left hand side of the above identity can be factored into the form:

$$
\left(\Delta^{2}-\lambda^{2}\right)\left(-\frac{1}{\lambda^{2}}\right)\left(1+\frac{\Delta^{2}}{\lambda^{2}}\right) r^{3}=r^{3}
$$

Comparing (2.40) and (2.44), we have

$$
\Phi^{(2)}(r)=\left(-\frac{1}{\lambda^{2}}\right)\left(1+\frac{\Delta^{2}}{\lambda^{2}}\right) r^{3}=-\frac{r^{3}}{\lambda^{2}}-\frac{24}{\lambda^{4} r^{4}}, \quad \text { for } r \neq 0 .
$$


Notice again we need to pay special attention to the singularity at $r=0$. We observe that

$$
\begin{array}{ll}
\Delta\left(\frac{e^{-\sqrt{\lambda} r}}{r}\right)=\frac{\lambda}{r} e^{-\sqrt{\lambda} r}, & \Delta^{2}\left(\frac{e^{-\sqrt{\lambda} r}}{r}\right)=\frac{\lambda^{2}}{r} e^{-\sqrt{\lambda} r}, \\
\Delta\left(\frac{\cos (\sqrt{\lambda} r)}{r}\right)=-\frac{\lambda}{r} \cos (\sqrt{\lambda} r), & \Delta^{2}\left(\frac{\cos (\sqrt{\lambda} r)}{r}\right)=\frac{\lambda^{2}}{r} \cos (\sqrt{\lambda} r) .
\end{array}
$$

Then,

$$
\left(\Delta^{2}-\lambda^{2}\right) \frac{e^{-\sqrt{\lambda} r}}{r}=0, \quad\left(\Delta^{2}-\lambda^{2}\right) \frac{\cos (\sqrt{\lambda} r)}{r}=0 .
$$

This implies that we can add any multiples of $e^{-\sqrt{\lambda} r} / r$ and $\cos (\sqrt{\lambda} r) / r$ to $\Phi^{(2)}(r)$ in (2.45) and $\Phi^{(2)}(r)$ is still a particular solution. As a result, we assume

$$
\Phi^{(2)}(r)=-\frac{r^{3}}{\lambda^{2}}-\frac{24}{r \lambda^{4}}+a \frac{e^{-\sqrt{\lambda} r}}{r}+b \frac{\cos (\sqrt{\lambda} r)}{r} .
$$

Then, it follows that

$$
\begin{aligned}
\frac{1}{r} \frac{\partial \Phi^{(2)}(r)}{\partial r}=-\frac{3 r}{\lambda^{2}} & +\frac{24}{\lambda^{4} r^{3}}+a\left(-\sqrt{\lambda} \frac{e^{-\sqrt{\lambda} r}}{r^{2}}-\frac{e^{-\sqrt{\lambda} r}}{r^{3}}\right) \\
& +b\left(-\sqrt{\lambda} \frac{\sin (\sqrt{\lambda} r)}{r^{2}}-\frac{\cos (\sqrt{\lambda} r)}{r^{3}}\right),
\end{aligned}
$$

and

$$
\Delta \Phi^{(2)}(r)=-\frac{12 r}{\lambda^{2}}+a \lambda \frac{e^{-\sqrt{\lambda} r}}{r}+b(-\lambda) \frac{\cos (\sqrt{\lambda} r)}{r} .
$$

By Taylor series expansion, we have the following expressions

$$
\begin{aligned}
& e^{-\sqrt{\lambda} r}=1-\sqrt{\lambda} r+\frac{1}{2} \lambda r^{2}-\frac{1}{6} \lambda^{\frac{3}{2}} r^{3}+\mathcal{O}\left(r^{4}\right), \\
& \cos (\sqrt{\lambda} r)=1-\frac{1}{2} \lambda r^{2}+\frac{1}{24} \lambda^{2} r^{4}+\mathcal{O}\left(r^{6}\right), \\
& \sin (\sqrt{\lambda} r)=\sqrt{\lambda} r-\frac{1}{6} \lambda^{\frac{3}{2}} r^{3}+\mathcal{O}\left(r^{5}\right) .
\end{aligned}
$$

From (2.49)-(2.51), to remove the singularity of $1 / r$ in (2.46), we have

$$
-\frac{24}{\lambda^{4}}+a+b=0 .
$$

To remove the singularities of $1 / r, 1 / r^{2}$ and $1 / r^{3}$ in (2.47), we have that

$$
\begin{aligned}
& a(-\sqrt{\lambda})(-\sqrt{\lambda})+a(-1) \frac{\lambda}{2}-b \lambda+b(-1)\left(-\frac{\lambda}{2}\right)=0, \\
& a(-\sqrt{\lambda})+a(-1)(-\sqrt{\lambda})=0, \\
& \frac{24}{\lambda^{4}}-a-b=0 .
\end{aligned}
$$


To remove the singularity of $1 / r$ in (2.48), we have

$$
\frac{a \lambda}{2}-\frac{b \lambda}{2}=0
$$

Notes that (2.52) and (2.55) are identical and (2.54) is true for any value $a$. Hence, (2.52)-(2.56) have only one solution which is

$$
a=b=\frac{12}{\lambda^{4}} .
$$

Thus,

$$
\Phi^{(2)}(r)= \begin{cases}\frac{-r^{3}}{\lambda^{2}}-\frac{24}{r \lambda^{4}}+\frac{12}{\lambda^{4} r}\left(e^{-\sqrt{\lambda} r}+\cos (\sqrt{\lambda} r)\right), & \text { for } r \neq 0, \\ -12 \lambda^{-\frac{7}{2}}, & \text { for } r=0 .\end{cases}
$$

Therefore, we have

$$
\frac{1}{r} \frac{\partial \Phi^{(2)}(r)}{\partial r}=\left\{\begin{array}{cc}
-\frac{3 r}{\lambda^{2}}+\frac{24}{\lambda^{4} r^{3}}-\frac{12 \sqrt{\lambda}}{\lambda^{4} r^{2}}\left(e^{-\sqrt{\lambda} r}+\sin (\sqrt{\lambda} r)\right) & \\
-\frac{12}{\lambda^{4} r^{3}}\left(e^{-\sqrt{\lambda} r}+\cos (\sqrt{\lambda} r)\right), & \text { for } r \neq 0 \\
-4 \lambda^{-\frac{5}{2}}, & \text { for } r=0
\end{array}\right.
$$

and

$$
\Delta \Phi^{(2)}(r)= \begin{cases}-\frac{12 r}{\lambda^{2}}+\frac{12}{\lambda^{3} r}\left(e^{-\sqrt{\lambda} r}-\cos (\sqrt{\lambda} r)\right), & \text { for } r \neq 0, \\ -12 \lambda^{-\frac{5}{2}}, & \text { for } r=0 .\end{cases}
$$

In general, we can obtain the general form of $\Phi^{(n)}(r)$ using the same procedure. Since

$$
\Delta^{m} r^{2 n-1}=0, \quad m=\left[\frac{n}{2}\right],
$$

it follows that

$$
\left(\Delta^{2}-\lambda^{2}\right)\left(-\frac{1}{\lambda^{2}}\right) \sum_{i=0}^{m}\left(\frac{\Delta^{2}}{\lambda^{2}}\right)^{i} r^{2 n-1}=r^{2 n-1}
$$

Thus,

$$
\Phi^{(n)}(r)=-\frac{1}{\lambda^{2}} \sum_{i=0}^{m}\left(\frac{\Delta^{2}}{\lambda^{2}}\right)^{i} r^{2 n-1}, \quad m=\left[\frac{n}{2}\right] .
$$

For $n$ even, a singularity will appear in $\Delta^{2 m} r^{2 n}$ which must be removed as in the $\Phi^{(2)}$ case. For $n$ odd, there is no singularity in $\Phi^{(n)}(r)$, but the normal derivative of $\Phi^{(n)}(r)$ will still have a singularity which also comes from

$$
\frac{1}{r} \frac{\partial \Delta^{2 m} r^{2 n}}{\partial r}
$$


It follows that if $r>0$,we can obtain the following

$$
\Phi^{(n)}(r)=-\frac{1}{\lambda^{2}} \sum_{i=0}^{m}\left(\frac{\Delta^{2}}{\lambda^{2}}\right)^{i} r^{2 n-1}+\frac{(2 n) !}{2 \lambda^{n+2 r}}\left(e^{-\sqrt{\lambda} r}+(-1)^{n} \cos (\sqrt{\lambda} r)\right),
$$

and

$$
\Phi^{(n)}(0)=\lim _{r \rightarrow 0} \Phi^{n}(r) .
$$

If the differential operator $\left(\Delta^{2}-\lambda^{2}\right)$ in (2.40) is replaced by $\left(\Delta^{2}+\lambda^{2}\right)$, a similar result follows:

$$
\Phi^{(n)}(r)=\frac{1}{\lambda^{2}} \sum_{i=0}^{m}\left(-\frac{\Delta^{2}}{\lambda^{2}}\right)^{i} r^{2 n+1}+\frac{(-1)^{m+1}(2 n) !}{2 \lambda^{n+2} r}\left(e^{-\sqrt{\lambda} r}+(-1)^{n} \cos (\sqrt{\lambda} r)\right) .
$$

\section{Particular solution for monomial term}

Next, we need to find the closed-form particular solutions for (2.12). We consider particular solutions of the following equation with the monomial term as the forcing term.

$$
\left(\Delta^{2}-\lambda^{2}\right) \chi=x^{k} y^{l}
$$

where $k$ and $l$ are nonnegative integers. To find $\chi$, a similar derivation for the Chebyshev polynomial can be found in [3]. We start with the following identity

$$
\left(1-\left(\frac{\Delta^{2}}{\lambda^{2}}\right)^{L+1}\right) x^{k} y^{l}=x^{k} y^{l}
$$

where $L$ is sufficiently large. The left hand side of (3.2) can be factored as follows:

$$
\left(\Delta^{2}-\lambda^{2}\right)\left(\frac{-1}{\lambda^{2}}\right) \sum_{i=0}^{L}\left(\frac{\Delta^{2}}{\lambda^{2}}\right)^{i} x^{k} y^{l}=x^{k} y^{l}
$$

Again, comparing (3.3) with (3.1), we obtain

$$
\chi=\frac{-1}{\lambda^{2}} \sum_{i=0}^{L}\left(\frac{\Delta^{2}}{\lambda^{2}}\right)^{i} x^{k} y^{l} .
$$

A similar conclusion can be obtained for the case $\Delta^{2}+\lambda^{2}$ in the 3D case. For illustration, we consider the following equation

$$
\left(\Delta^{2}-\lambda^{2}\right) \chi=x^{3} y^{2} .
$$

Using (3.4), we obtain

$$
\chi=\frac{-1}{\lambda^{2}}\left(1+\frac{\Delta^{2}}{\lambda^{2}}+\frac{\Delta^{4}}{\lambda^{4}}+\ldots\right) x^{3} y^{2}=\frac{-x^{3} y^{2}}{\lambda^{2}} .
$$




\section{Numerical examples}

To validate the efficacy of the proposed method, we consider three examples in this section. All of the numerical results compare well with the analytical solutions. We will examine the influence of the number of boundary, interior nodes, and order of polyharmonic splines on the accuracy of numerical results.

The root-mean-square error (RMSE) and the root-mean-square error of the derivative with respect to $x$ (RMSEx) are used in this section to demonstrate the accuracy of the solutions and they are defined as follows:

$$
\begin{aligned}
& \text { RMSE }=\sqrt{\frac{1}{q} \sum_{j=1}^{q}\left(\widehat{u}_{j}-u_{j}\right)^{2}}, \\
& \text { RMSEx }=\sqrt{\frac{1}{q} \sum_{j=1}^{q}\left(\frac{\partial \widehat{u}_{j}}{\partial x}-\frac{\partial u_{j}}{\partial x}\right)^{2}},
\end{aligned}
$$

where $q$ is the number of testing nodes located uniformly within the domain. $\widehat{u}_{j}$ denotes the numerical solution at the $j^{\text {th }}$ node. According to the following numerical tests, we found that the RMSEy is similar to RMSEx. Therefore, we only show the $R M S E$ and RMSEx in the following tests.

To approximate the homogeneous solution, we apply the method of fundamental solutions (MFS) $[8,9]$. In the MFS, the source points are located on the fictitious boundary outside the computational domain. There are various ways to determine the location of source points. In this paper, we apply the following approach to determine how to place the source points

$$
\mathbf{x}^{s}=\mathbf{x}^{b}+d\left(\mathbf{x}^{b}-\mathbf{x}^{c}\right),
$$

where $\mathbf{x}^{s}, \mathbf{x}^{b}$ and $\mathbf{x}^{c}$ denote the source, boundary, and central nodes. $d$ determines how far away of the source points to the boundary. The search of optimal $d$ is still an outstanding research problem. In the following examples, we find little difference using different values of $d$.

Example 1. Consider the following problem in thin plate vibration

$$
\begin{array}{ll}
\left(\Delta^{2}-2000\right) u(x, y)=f(x, y), & (x, y) \in \Omega, \\
u(x, y)=\sin \left(y^{2}+x\right)-\cos \left(y-x^{2}\right), & (x, y) \in \partial \Omega \\
\frac{\partial u(x, y)}{\partial \mathbf{n}}=\nabla\left(\sin \left(y^{2}+x\right)-\cos \left(y-x^{2}\right)\right) \cdot \mathbf{n}, & (x, y) \in \partial \Omega
\end{array}
$$

where $\mathbf{n}$ is the normal vector along the boundary.

The non-homogeneous term $f(x, y)$ is given based on the analytical solution, which is as follows:

$$
u(x, y)=\sin \left(y^{2}+x\right)-\cos \left(y-x^{2}\right), \quad(x, y) \in \Omega .
$$


Table 1: RMSE and RMSEx obtained by different number of boundary nodes $n_{b}$, interpolation nodes $n_{i}$ and order of polyharmonic splines $n$.

\begin{tabular}{||lllll||}
\hline & $n_{b}$ & 80 & 120 & 160 \\
& $n_{i}$ & 101 & 202 & 318 \\
\hline$n=1$ & RMSE & $1.33 E-3$ & $3.73 E-4$ & $1.43 E-4$ \\
& RMSEx & $1.09 E-2$ & $3.35 E-3$ & $1.62 E-3$ \\
\hline$n=2$ & RMSE & $1.94 E-4$ & $1.67 E-5$ & $1.02 E-5$ \\
& RMSEx & $1.47 E-3$ & $1.81 E-4$ & $9.21 E-5$ \\
\hline$n=3$ & RMSE & $6.23 E-4$ & $2.48 E-5$ & $4.05 E-6$ \\
& RMSEx & $4.76 E-3$ & $2.74 E-4$ & $4.69 E-5$ \\
\hline
\end{tabular}
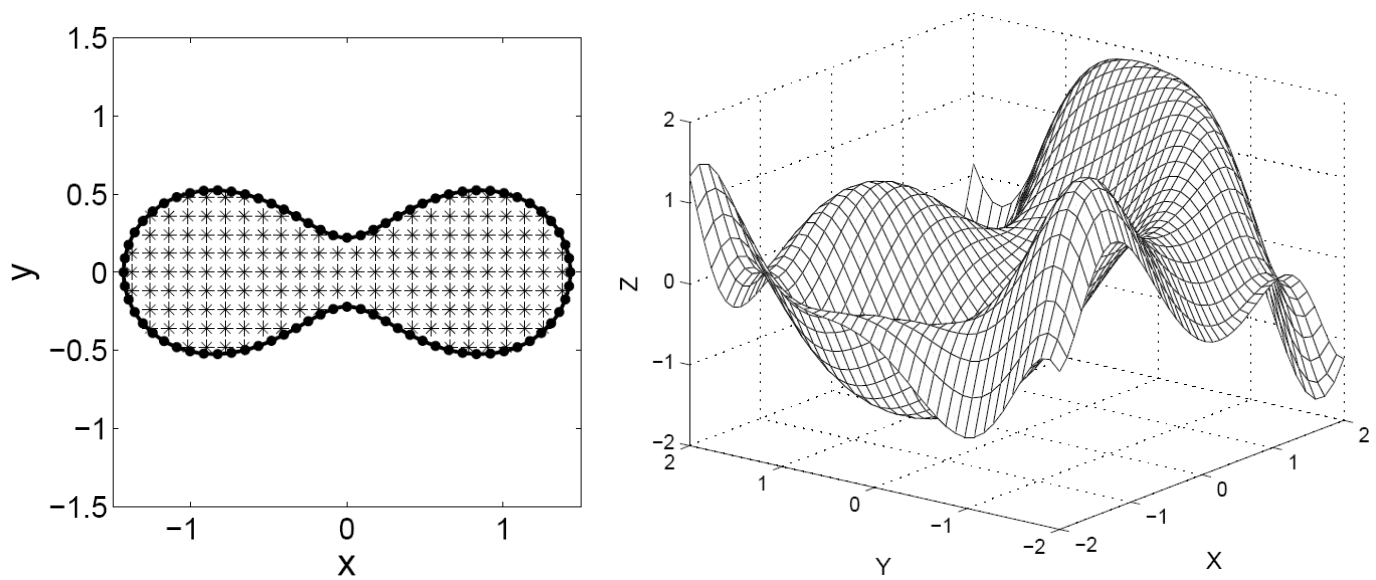

Figure 1: Interior points $(*)$ and boundary points $(\bullet)$ (left) and the profile of solution in the extended domain.

The computational domain is defined by the following parametric equation:

$$
\Omega=\{(x, y) \mid x=\rho \cos \theta, \quad y=\rho \sin \theta, \quad 0 \leq \theta \leq 2 \pi\},
$$

where

$$
\rho=\left(\cos (2 \theta)+\sqrt{1.1-\sin ^{2}(2 \theta)}\right) .
$$

The computational domain, distribution of interior and boundary nodes, and the profile of solution in the extended domain are shown in Fig. 1. The number of testing nodes $q=212$ is used for calculating the RMSE and RMSEx. Note that at each boundary nodes there are two boundary conditions imposed. Hence, we have an overdetermined system of equations. In this case, we have to use the linear least-square solver. The RMSE and RMSEx are shown in Table 1 where the number of boundary nodes and interior nodes are chosen as $(80,101),(120,202)$, and $(160,318)$. The order of the polyharmonic splines $\left(r^{2 n} \ln r\right)$ is used for $n=1,2,3$. To obtain the homogeneous solution, we applied the MFS. To locate the source points, we choose $d=6$.

As shown in Table 1, the results improved with the increases of the number of boundary, interior nodes, and the order of polyharmonic splines. 
Example 2. In this example, we consider the similar problem as the previous example with different boundary conditions and domain

$$
\begin{array}{ll}
\left(\Delta^{2}-100\right) u(x, y)=f(x, y), & (x, y) \in \Omega, \\
u(x, y)=\sin (\pi x) \cosh (y)-\cos (\pi x) \sinh (y), & (x, y) \in \partial \Omega, \\
\Delta u(x, y)=\Delta(\sin (\pi x) \cosh (y)-\cos (\pi x) \sinh (y)), & (x, y) \in \partial \Omega .
\end{array}
$$

The non-homogeneous term $f(x, y)$ is based on the analytical solution, which is shown as follows:

$$
u(x, y)=\sin (\pi x) \cosh (y)-\cos (\pi x) \sinh (y), \quad(x, y) \in \Omega
$$

The computational domain is defined by the following parametric equation:

$$
\Omega=\{(x, y) \mid x=\rho \cos \theta, y=\rho \sin \theta, \quad 0 \leq \theta \leq 2 \pi\},
$$

where

$$
\rho=\left(\cos (3 \theta)+\sqrt{2-\sin ^{2}(3 \theta)}\right)^{\frac{1}{3}} .
$$

The computational domain, distribution of nodes, and profile of solution are shown in Fig. 2. We choose $q=230$ testing nodes for calculating the RMSE and RMSEx. Similar to the previous example, we use the linear least-square solver to solve the overdetermined system. To compute RMSE and RMSEx, the number of boundary and interior nodes are chosen as $(80,126),(140,280)$ and $(200,374)$ with the order of the ployharmonic splines $n=1,2,3$. For the MFS, we choose $d=4$.

From Table 2, we observe that even with a small amount of boundary and interior nodes, we can still achieve good accuracy. This further confirms that once we obtain the closed-form particular solution and fundamental solution of the given differential equation, the problem can be solved efficiently.

Table 2: RMSE and RMSEx obtained by different number of boundary nodes, $n_{b}$, interpolation nodes, $n_{i}$, and order of polyharmonic splines, $n$.

\begin{tabular}{||lllll||}
\hline & $n_{b}$ & 80 & 140 & 200 \\
& $n_{i}$ & 126 & 208 & 374 \\
\hline$n=1$ & RMSE & $7.34 E-5$ & $3.31 E-5$ & $4.16 E-5$ \\
& RMSEx & $7.20 E-4$ & $4.36 E-4$ & $4.19 E-4$ \\
\hline$n=2$ & RMSE & $4.52 E-5$ & $1.58 E-5$ & $7.84 E-6$ \\
& RMSEx & $2.04 E-4$ & $1.32 E-4$ & $8.39 E-5$ \\
\hline$n=3$ & RMSE & $2.44 E-5$ & $5.89 E-6$ & $1.64 E-6$ \\
& RMSEx & $8.59 E-5$ & $3.48 E-5$ & $1.71 E-5$ \\
\hline
\end{tabular}



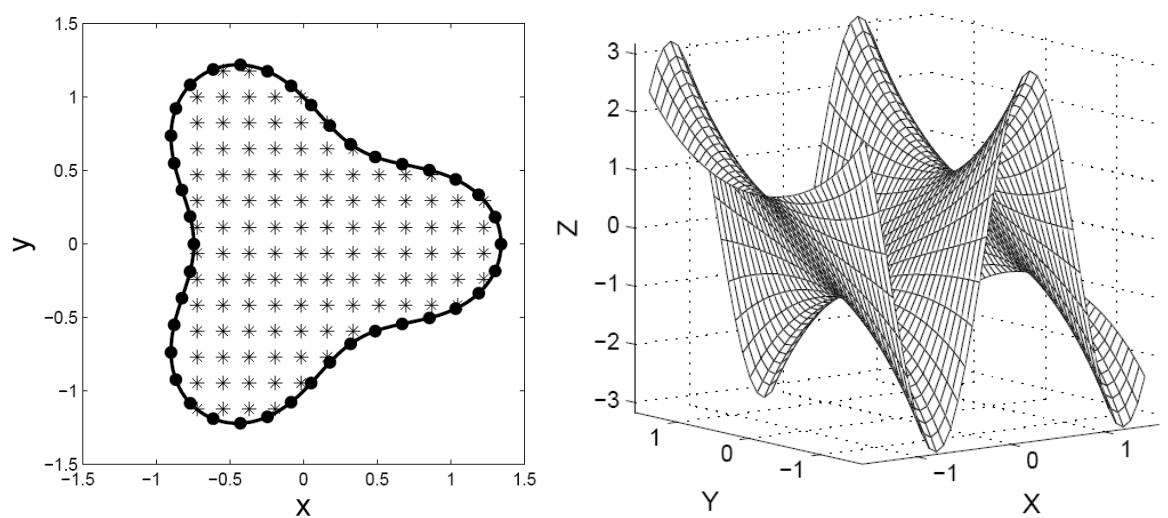

Figure 2: Computational domain (left) and profile of solution (right) in the extended domain.

Example 3. In this example, we consider the three dimensional problem

$$
\begin{array}{ll}
\left(\Delta^{2}-1000\right) u(x, y, z)=f(x, y, z), & (x, y, z) \in \Omega, \\
u(x, y, z)=\frac{1}{120}\left(x^{5}+y^{5}+z^{5}\right), & (x, y, z) \in \partial \Omega, \\
\Delta u(x, y, z)=g(x, y, z), & (x, y, z) \in \partial \Omega,
\end{array}
$$

where $f(x, y, z)$ and $g(x, y, z)$ are based on the analytical solution, which is shown as follows:

$$
u(x, y, z)=\frac{1}{120}\left(x^{5}+y^{5}+z^{5}\right), \quad(x, y, z) \in \Omega .
$$

The computational domain, unit sphere, and distribution of boundary nodes are shown in Fig. 3. We choose $q=93$ randomly distributed interior testing nodes for calculating the RMSE and RMSEx. Note that the number of boundary and interior nodes are chosen as $(20,27),(80,93),(140,160),(200,251)$ and $(260,360)$ with the order of the polyharmonic splines $n=1,2,3,4$. The numerical results are shown in Tables

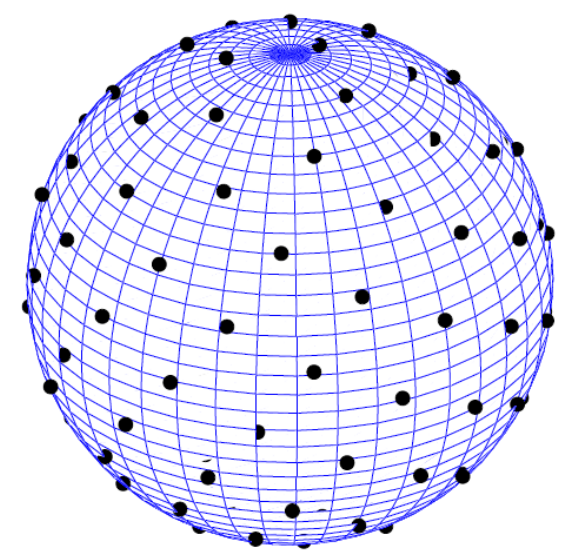

Figure 3: Computational domain and boundary points. 
Table 3: RMSE error using various order of polyharmonic spliens.

\begin{tabular}{||llllll||}
\hline$n_{b}$ & 20 & 80 & 140 & 200 & 260 \\
$n_{i}$ & 27 & 93 & 160 & 251 & 360 \\
\hline$n$ & \multicolumn{5}{l}{ RMSE } \\
\hline 1 & $1.23 E-3$ & $8.96 E-4$ & $9.20 E-4$ & $9.77 E-4$ & $1.14 E-3$ \\
2 & $1.27 E-3$ & $8.37 E-4$ & $9.19 E-4$ & $9.96 E-4$ & $1.07 E-3$ \\
3 & $1.20 E-3$ & $6.76 E-4$ & $5.01 E-4$ & $4.12 E-4$ & $3.11 E-4$ \\
4 & $1.88 E-3$ & $7.77 E-4$ & $3.17 E-4$ & $2.65 E-4$ & $2.15 E-4$ \\
\hline
\end{tabular}

Table 4: RMSE errors using various order of polyharmonic splines.

\begin{tabular}{||llllll||}
\hline$n_{b}$ & 20 & 80 & 140 & 200 & 260 \\
$n_{i}$ & 27 & 93 & 160 & 251 & 360 \\
\hline$n$ & & & $R M S E x$ \\
\hline 1 & $4.35 E-3$ & $3.24 E-3$ & $2.74 E-3$ & $2.34 E-3$ & $2.47 E-3$ \\
2 & $4.51 E-3$ & $2.40 E-3$ & $2.10 E-3$ & $2.20 E-3$ & $2.41 E-3$ \\
3 & $4.30 E-3$ & $2.05 E-3$ & $1.37 E-3$ & $1.12 E-3$ & $9.18 E-4$ \\
4 & $6.43 E-3$ & $2.51 E-3$ & $1.33 E-3$ & $9.70 E-4$ & $8.76 E-4$ \\
\hline
\end{tabular}

Table 5: The optimal distance $d$ between boundary and source points corresponding Tables 3 and 4 .

\begin{tabular}{||llllll||}
\hline$n_{b}$ & 20 & 80 & 140 & 200 & 260 \\
$n_{i}$ & 27 & 93 & 160 & 251 & 360 \\
\hline$n$ & \multicolumn{5}{c||}{$d_{\text {opt }}$} \\
\hline 1 & 1.0765 & 1.0254 & 1.0097 & 1.0061 & 1.0042 \\
2 & 1.0796 & 1.0161 & 1.0051 & 1.0019 & 1.0005 \\
3 & 1.0769 & 1.0146 & 1.0076 & 1.0048 & 1.0068 \\
4 & 1.0811 & 1.0178 & 1.0157 & 1.0124 & 1.0193 \\
\hline
\end{tabular}

3 and 4 . When we increase the order of polyharmonic spline and the number of boundary points and interior points, the RMSE and RMSEx become smaller. The optimal $d$ for the source points in the MFS corresponding to Tables 3 and 4 are shown in Table 5 . Since our main focus in this paper is the derivation of particular solutions, we will not further elaborate how we choose the optimal $d$.

In general, it is nontrivial to solve a 3D problem using traditional numerical methods due to the complication of domain and surface meshing. In our approach, once particular solutions are derived, a 3D problem can be handled as easily as a 2D problem.

\section{Conclusions}

Using a very simple algebraic factorization approach, we derive a closed-form particular solution in which polyharmonic splines have been used as basis functions to approximate the non-homogeneous term of a given differential operator of the form 
$\Delta^{2} \pm \lambda^{2}$. Instead of tedious derivation of particular solutions as shown in [12,13], our derivation of particular solutions is simple and direct. Our approach for the derivation of particular solutions can be easily extended to product of poly-Helmholtz operators.

\section{Acknowledgement}

The authors thank Dr. C. M. Fan for his helpful comments and suggestions.

\section{Appendix}

In the $2 \mathrm{D}$ case, for $n=3,4$, particular solutions for (2.13) is given by

$$
\begin{aligned}
& \Phi^{(3)}(r)= \begin{cases}-\frac{r^{6} \ln r}{\lambda^{2}}-\frac{r^{2}}{\lambda^{4}}(576 \ln r+480)-\frac{576}{\lambda^{5}}\left(2 K_{0}(\sqrt{\lambda} r)+\pi Y_{0}(\sqrt{\lambda} r)\right), & \text { for } r \neq 0, \\
0, & \text { for } r=0 .\end{cases} \\
& \frac{1}{r} \frac{\partial \Phi^{(3)}(r)}{\partial r}=\left\{\begin{array}{rlrl}
-\frac{r^{4}}{\lambda^{2}}(6 \ln r+1) & -\frac{384}{\lambda^{4}}(3 \ln r+4) & \\
& +\frac{576 \sqrt{\lambda}}{\lambda^{5} r}\left(2 K_{1}(\sqrt{\lambda} r)+\pi Y_{1}(\sqrt{\lambda} r)\right), & \text { for } r \neq 0 \\
\frac{192}{\lambda^{4}}\left(11-6 \gamma-6 \ln \left(\frac{\sqrt{\lambda}}{2}\right)\right), & \text { for } r=0 .
\end{array}\right. \\
& \Delta \Phi^{(3)}(r)=\left\{\begin{array}{rr}
-\frac{384}{\lambda^{4}}(6 \ln r+11)-\frac{12 r^{4}}{\lambda^{2}}(3 \ln r+1) & \\
-\frac{576}{\lambda^{4}}\left(2 K_{0}(\sqrt{\lambda} r)-\pi Y_{0}(\sqrt{\lambda} r)\right), & \text { for } r \neq 0, \\
-\frac{384}{\lambda^{4}}\left(11-6 \gamma-6 \ln \left(\frac{\sqrt{\lambda}}{2}\right)\right), & \text { for } r=0 .
\end{array}\right. \\
& \Phi^{(4)}(r)=\left\{\begin{array}{cl}
-\frac{r^{8} \ln r}{\lambda^{2}}-\frac{192 r^{4}}{\lambda^{4}}(12 \ln r+7)-\frac{12288}{\lambda^{6}}(12 \ln r+25) & \\
-\frac{36864}{\lambda^{6}}\left(2 K_{0}(\sqrt{\lambda} r)-\pi Y_{0}(\sqrt{\lambda} r)\right), & \text { for } r \neq 0, \\
\frac{12288}{\lambda^{6}}\left(-25+12 \gamma+12 \ln \left(\frac{\sqrt{\lambda}}{2}\right)\right), & \text { for } r=0 .
\end{array}\right.
\end{aligned}
$$

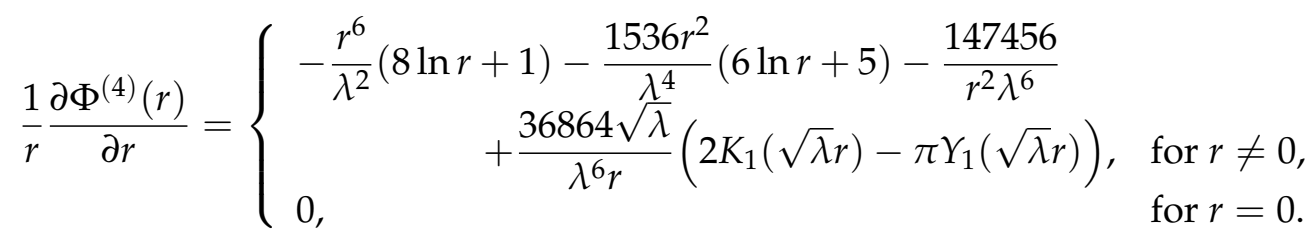




$$
\Delta \Phi^{(4)}(r)=\left\{\begin{array}{cc}
-\frac{16 r^{6}}{\lambda^{4}}(4 \ln r+1)-\frac{3072 r^{2}}{\lambda^{4}}(12 \ln r+13) & \\
-\frac{36864}{\lambda^{5}}\left(2 K_{0}(\sqrt{\lambda} r)+\pi Y_{0}(\sqrt{\lambda} r)\right), & \text { for } r \neq 0, \\
-\frac{384}{\lambda^{4}}\left(11-6 \gamma-6 \ln \left(\frac{\sqrt{\lambda}}{2}\right)\right), & \text { for } r=0 .
\end{array}\right.
$$

In the 3D case, for $n=3,4$, particular solutions for (2.13) is given by

$$
\begin{aligned}
& \Phi^{(3)}(r)= \begin{cases}-\frac{r^{5}}{\lambda^{2}}-\frac{360 r}{\lambda^{4}}+\frac{360}{\lambda^{5} r}\left(e^{-\sqrt{\lambda} r}-\cos (\sqrt{\lambda} r)\right), & \text { for } r \neq 0, \\
-360 \lambda^{-\frac{9}{2}}, & \text { for } r=0,\end{cases} \\
& \frac{1}{r} \frac{\partial \Phi^{(3)}(r)}{\partial r}=\left\{\begin{array}{cc}
-\frac{5 r^{3}}{\lambda^{2}}-\frac{360 \sqrt{\lambda}}{\lambda^{5} r^{2}}\left(e^{-\sqrt{\lambda} r}-\sin (\sqrt{\lambda} r)\right) & \\
-\frac{360}{r \lambda^{4}}-\frac{360}{\lambda^{5} r^{3}}\left(e^{-\sqrt{\lambda} r}-\cos (\sqrt{\lambda} r)\right), & \text { for } r \neq 0, \\
-120 \lambda^{-\frac{7}{2}}, & \text { for } r=0,
\end{array}\right. \\
& \Delta \Phi^{(3)}(r)= \begin{cases}-\frac{30 r^{3}}{\lambda^{2}}-\frac{720}{r \lambda^{4}}+\frac{360}{\lambda^{4} r}\left(e^{-\sqrt{\lambda} r}+\cos (\sqrt{\lambda} r)\right), & \text { for } r \neq 0, \\
-360 \lambda^{-\frac{7}{2}}, & \text { for } r=0 .\end{cases} \\
& \Phi^{(4)}(r)= \begin{cases}-\frac{r^{7}}{\lambda^{2}}-\frac{1680 r^{3}}{\lambda^{4}}-\frac{40320}{r \lambda^{6}}+\frac{20160}{\lambda^{6} r}\left(e^{-\sqrt{\lambda} r}+\cos (\sqrt{\lambda} r)\right), & \text { for } r \neq 0 \\
-20160 \lambda^{-\frac{11}{2}} & \text { for } r=0 .\end{cases}
\end{aligned}
$$

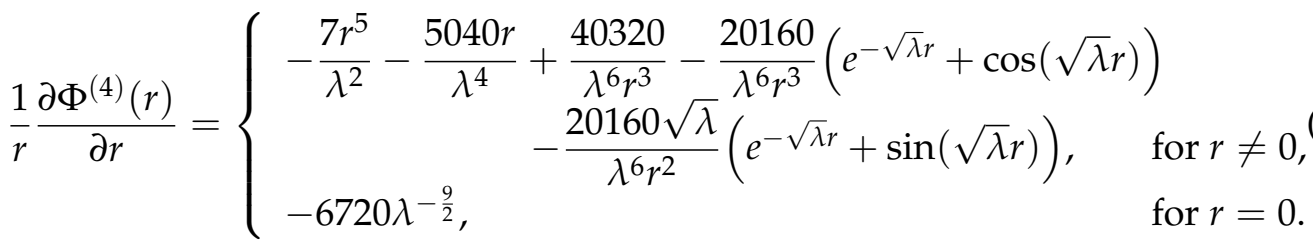

$$
\begin{aligned}
& \Delta \Phi^{(4)}(r)= \begin{cases}-\frac{56 r^{5}}{\lambda^{2}}-\frac{20160 r}{\lambda^{4}}+\frac{20160}{\lambda^{5} r}\left(e^{-\sqrt{\lambda} r}-\cos (\sqrt{\lambda} r)\right), & \text { for } r \neq 0 \\
-20160 \lambda^{-\frac{9}{2}}, & \text { for } r=0 .\end{cases}
\end{aligned}
$$

\section{References}

[1] H. A. Cho, M. A. Golberg, A. S. Muleshrov And X. Li, Trefftz methods for time dependent partial differential equations, Computers, Materials and Continua., 1 (2004), pp. $1-37$.

[2] C. S. CHEN AND Y. F. RASHED, Evaluation of thin plate spline based particular solutions for Helmholtz-type operators for the DRM, Mech. Res. Commun., 25 (1998), pp. 195-201. 
[3] C. S. Chen, S. LeE AND C. S. HuANG, Derivation of particular solutions using Chebyshev polynomial based functions, Int. J. Comput. Meth., 4 (2007), pp. 15-32.

[4] W. CHEN, Symmetric boundary knot method, Eng. Anal. Bound. Elem., 26 (2002), pp. 489494.

[5] W. CHEN, Z. J. SHeN, L. J. SHEN AND G. W. YUAN, General solutions and fundamental solutions of varied orders to the vibrational thin, the Berge, and the Winkler plates, Eng. Anal. Bound. Elem., 29 (2005), pp. 699-702.

[6] A. H. D. CHENG, Particular solutions of Laplacian, Helmholtz-type, and polyharmonic operators involving higher order radial basis functions, Eng. Anal. Bound. Elem., 24 (2000), pp. 531-538.

[7] J. DuCHON, Sur l'erreur d interpolation des fonctions de plusiers variables par les $D^{m}$-splines, RAIRO Anal. Numer., 12 (1978), pp. 325-334.

[8] G. FAIRWEATHER AND A. KARAGEORGHIS, The method of fundamental solutions for elliptic boundary value problems, Adv. Comput. Math., 9 (1998), pp. 69-95.

[9] M. A. Golberg AND C. S. CHEN, The method of fundamental solutions for potential, Helmholtz and diffusion problems, in Boundary Integral Methods - Numerical and Mathematical Aspects, ed. M.A. Golberg, Computational Mechanics Publications, (1998), pp. 103-176.

[10] A. Milton AND A. S. IREne, Handbook of Mathematical Functions with Formulas, Graphs and Mathematical Tables, Dover, New York, (1964).

[11] A. S. Muleshrov, M. A. Golberg AND C. S. Chen, Particular solutions of Helmholtztype operators using higher order polyharmonic splines, Comput. Mech., 24 (1999), pp. 411419.

[12] A. S. MuleshKov And M. A. GolberG, Particular solutions of the multi-Helmholtz-type equation, Eng. Anal. Bound. Elem., 31 (2007), pp. 624-630.

[13] C. C. TSAI, A. H. D. CHENG AND C. S. CHEN, Particular solutions of splines and monomials for polyharmonic and products of Helmholtz operators, Eng. Anal. Bound. Elem., 33 (2009), pp. 514-521.

[14] D. L. YounG, C. C. TSAI, Y. C. LIN AND C. S. CHEN, The method of fundamental solutions for eigenfrequencies of plate vibrations, J. Comp. Mater. Con., 4 (2006), pp. 1-10. 